\title{
Les relations entre les enseignants et les parents : premières étapes d'intervention d'un Laboratoire du Changement
}

\author{
Rollande Deslandes* et Sylvie Barma** \\ DOI https://doi.org/10.51657/ric.v3i1.41025
}

\begin{abstract}
Résumé La présente recherche intervention vise à illustrer les premières étapes du processus d'un Laboratoire du Changement, une méthode de recherche et d'intervention formative et encore peu connue au Québec, appliquée ici dans le contexte des relations enseignants-parents. Nous désirons mettre à profit cette méthode dans un contexte de réforme curriculaire difficile. L'objet ultime du Laboratoire du Changement est de promouvoir la participation parentale au suivi scolaire par l'entremise de l'amélioration des relations entre les enseignants et les parents. Les études réalisées depuis quelques décennies montrent les bénéfices associés à des relations enseignants-parents positives et collaboratrices pour la réussite scolaire. De ces relations émergent des zones de tensions exprimées sous la forme de manifestations discursives de contradictions. À la lumière de la théorie de l'activité et de la méthode du Laboratoire du Changement, l'analyse de deux premières rencontres réunissant neuf participants a mené à l'identification des contradictions primaires à chacun des pôles du triangle de l'activité. Celles-ci ont trait surtout à la volonté ou non de favoriser une plus grande participation parentale (objet), à des communications parfois ambiguës (outil), à des attentes irréalistes de certains parents à l'égard de rétroactions attendues de la part des enseignants (division du travail), à des façons de faire qui ne respectent pas les règles de fonctionnement de l'école, au caractère individualiste des rapports de certains parents avec les enseignants et de certains services offerts par des professionnels uniquement pendant les heures de classe (communauté). Cette analyse dialectique réalisée par les chercheurs a ainsi permis d'identifier les problèmes rencontrés et d'en discuter avec les participants. Les résultats de l'analyse des manifestations de ces contradictions seront réinvestis à titre de données miroir dans une phase ultérieure du Laboratoire du changement afin d'outrepasser ces problèmes et de s'engager dans une comodélisation d'une nouvelle forme de relation entre les enseignants et les parents.
\end{abstract}

Mots-clés relations parents-enseignants; Laboratoire du Changement; théorie de l'activité; participation parentale

\footnotetext{
* Centre de recherche et d'intervention pour la réussite scolaire (CRIRES), Université du Québec à Trois-Rivières

** Centre de recherche et d'intervention pour la réussite scolaire (CRIRES), Université Laval
} 


\section{Problématique et cadre de référence}

Au cours des trente dernières années s'est développé un consensus sur l'importance de relations école-famille collaboratrices. Ce rapprochement entre les divers milieux de vie des jeunes est devenu impérieux compte tenu des nombreux changements démographiques qui touchent les familles, de l'accroissement de l'écart entre les riches et les pauvres, des exigences des milieux de travail, de la diversité ainsi que de la complexité grandissante des problèmes des jeunes et de leur famille. En clair, l'école ne peut pas suffire à la tâche en solo (Deslandes, 2013; Deslandes et Bertrand, 2001).

Or, certains faits sont maintenant bien établies (Deslandes, 2011). Ainsi, les parents sont incontestablement les premiers éducateurs de leur enfant. De plus, bien que les caractéristiques familiales (structure, scolarité et revenu des parents) influent sur la réussite scolaire et le développement global de l'enfant, un grand nombre d'études réalisées sur le plan national et international ont montré que les processus familiaux tels que les attitudes, les valeurs et les pratiques des parents dans l'éducation de leur enfant sont encore plus importants (Christenson et Sheridan, 2001; Deslandes et Bertrand, 2004, 2005; Hoover-Dempsey, Ice, et Whitaker, 2010). Le concept de participation parentale au suivi scolaire revêt un caractère multidimensionnel (Deslandes, 2005). D'après des parents, la participation parentale au suivi scolaire comporte deux dimensions : 1) la participation à domicile (supervision, suivi dans les travaux scolaires) et 2) la participation à l'école (retour d'appels téléphoniques; signature de l'agenda et de travaux; présence à des rencontres et bénévolat) (Deslandes et Bertrand, 2004, 2005). La participation au suivi scolaire à domicile a des impacts directs sur les résultats scolaires (Epstein, 2011). Cette participation peut se traduire par des comportements tels que guider, discuter, aider, encourager, enseigner aux enfants pour qu'ils développent des attitudes positives et des comportements proscolaires. Par ailleurs, lorsque des parents se rendent à l'école pour faire du bénévolat, participer à des ateliers, etc., ils sont davantage susceptibles d'apprendre de quelles manières ils peuvent engager leur enfant dans des activités d'apprentissage et comment ils peuvent obtenir de l'aide. Ils deviennent alors plus capables de développer une relation constructive avec les enseignants (Deslandes, 2010; Epstein, 2011).

De leur côté, les relations école-famille renvoient aux liens officiels et informels entre l'école et la famille. Les relations école-famille collaboratrices réfèrent aux responsabilités familiales et au rôle de l'école dans l'actualisation de la participation des parents au suivi scolaire (Deslandes, 2013). Les pratiques de l'école sont donc susceptibles d'influencer les pratiques familiales (Deslandes, 2001; Epstein, 2011). Ainsi, les parents participent davantage à la maison et à l'école lorsqu'ils perçoivent que cette dernière favorise leur collaboration (Deslandes, 2008; Deslandes, Bastien et Lemieux, 2006). Cette collaboration se développe en présence d'un partage des responsabilités, d'une confiance mutuelle et d'une communication ouverte entre les partenaires (Deslandes, 2010, 2013). Des relations école-famille collaboratrices reposent nécessairement sur des savoir-faire et des savoir-être essentiels (Deslandes, 2006). Pour être collaboratrices, les relations école-famille doivent de surcroît répondre aux besoins des familles en matière d'information, de soutien et de formation de même qu'elles doivent prendre en compte leurs différences socioéconomiques et culturelles, c'est-à-dire, leur diversité (Deslandes, 2012). Les relations collaboratrices constituent un facteur de protection contre le décrochage scolaire, entre autres, en milieu défavorisé et contribuent à la persévérance scolaire (Deslandes, 2007). Elles semblent en effet amoindrir les effets négatifs de la pauvreté au regard des résultats scolaires et des comportements inappropriés des enfants (Caspe, Lopez, et Wolos, 2007; Deslandes, 2010; Kreider, Caspe, Kennedy, et Weiss, 2007).

Ce contexte particulier constitue un terreau propice à l'émergence de tensions, voire au maintien d'un état d'équilibre précaire dans les relations entre enseignants et parents. Comme l'illustre Deslandes (2012) ces relations sont susceptibles d'être influencées par des facteurs associés aux parents et aux familles, à l'école et aux enseignants, et aux jeunes eux-mêmes ainsi que par des facteurs environnementaux reliés aux motivations des parents à participer au suivi scolaire et aux motivations des enseignants à promouvoir la participation parentale au suivi scolaire. Le modèle illustré montre des conditions facilitantes et contraignantes qui contribuent ou nuisent au développement de relations école-famille collaboratrices. Par exemple, les expériences 
scolaires antérieures des parents et celles des enseignants en matière de travail avec les parents, les croyances, les valeurs et la compréhension de leur rôle respectif et des attentes des uns à l'égard des autres, ainsi que leur sentiment de compétence, leurs habiletés de communication et la prise en compte du temps et de la disponibilité de chacun sont autant d'éléments qui interviennent sur la qualité des relations entre les enseignants et les parents (Deslandes, 2012; Hoover-Dempsey et al., 2010). S'ajoutent aussi le soutien de la direction de l'école, des collègues et le développement de liens de confiance entre les enseignants et les parents. Il n'est donc pas surprenant que ce travail de collaboration axé sur le partage des responsabilités entre les enseignants et les parents suscite parfois des tensions. De ces espaces non délimités par des frontières claires en matière de rôle et de responsabilité de chacun émergent des dilemmes, des malentendus, des maladresses qui se révèlent des manifestations de contradictions, ces dernières étant entendues comme des tensions récurrentes s'étant accumulées au fil du temps dans un milieu donné (Engeström et Sannino, 2011).

À la lumière de ce constat et de l'importance de prendre en considération le fait que toute collaboration fait appel à la mise en place de convergences entre les différents acteurs impliqués, et ce, malgré l'émergence de nombreuses tensions, il semble opportun d'entreprendre une démarche de transformation individuelle et collective au sein d'un établissement scolaire désireux de développer dans ce sens. Pour ce faire, nous voulons mettre à profit un cadre théorique et méthodologique ancré dans la théorie de l'activité qui s'intéresse à la transformation des pratiques sociales (Barma, 2014). Ce cadre vise justement à documenter la transformation collective et la mise en place d'innovations dans un milieu donné (Barma, 2012; Engeström, 2015). La prochaine section développe les principales caractéristiques du Laboratoire du Changement, une méthode de recherche interventionniste qui tire son origine du Developmental Work Research (DWR) et qui fut développée en Finlande par Yrjö Engeström et son équipe depuis 1987. Cette approche d'analyse permet, dans un premier temps, l'identification de zones de tensions dans les relations entre les enseignants et les parents. Adaptée à la réalité des relations école-famille au Québec et au contexte culturel québécois, la théorie de l'activité permet ainsi de s'attarder à la façon dont les divers acteurs et les chercheurs concernés par la problématique des relations école-famille peuvent en arriver à modéliser conjointement des pistes de solution. Celles-ci sont mises en œuvre à la lumière de l'identification de problèmes rencontrés dans leur milieu, et ce, pour résoudre les tensions présentes sans toutefois s'immiscer dans l'espace de l'autre.

Deux rencontres en mode Labo ont été conduites. Les deux questions de recherche auxquelles nous avons tenté de répondre sont : 1) quelles sont les zones de tensions relevées par les participants dans les relations entre les enseignants et les parents? 2) quelles contradictions ont émergé lors des échanges entre les participants? L'analyse des deux rencontres avec les participants au Laboratoire du Changement est susceptible de mener à l'identification des motivations des acteurs concernés à s'engager dans une démarche de transformation individuelle et collective. Les sections qui suivent décrivent les aspects propres au cadre d'intervention et d'analyse choisi pour amorcer et documenter les premières étapes du Laboratoire du Changement mis en œuvre.

\section{Cadre d'intervention et d'analyse}

\section{La théorie de l'activité}

Les objectifs du Laboratoire du Changement initié dans le cadre de cette rechercheintervention visent à promouvoir de meilleures relations entre les enseignants et les parents dans le but de favoriser la participation des parents au suivi scolaire, et ce, dans une optique de meilleure réussite éducative du jeune. Dans le cadre de cet article, nous présentons d'abord le cadre d'intervention et d'analyse. Suit la description des deux premières rencontres du Laboratoire du Changement.

Prenant appui sur les travaux de Vygotsky (1978), Leont'ev (1978) et récemment d'Engeström et Sannino (2011), la troisième génération de la théorie de l'activité selon Engeström, (2001, 2007, 2010) permet d'entrevoir dans le présent cas une possible transformation des relations enseignants-parents (objet) dans une optique de plus grande participation parentale en vue d'une plus grande réussite scolaire de l'élève (résultat attendu). Les sujets (enseignants, 
parents, direction d'établissement) associés à des systèmes d'activité étudiés agissent en fonction d'un but commun et vivent des tensions qui résultent de règles établies dans leurs milieux respectifs et susceptibles d'en appeler à une clarification des rôles qui possiblement influencera la division du travail. Un système d'activité est toujours l'unité d'analyse. Il est orienté vers l'objet d'étude et médiatisé par des artéfacts comme par exemple, des outils, des instruments. Le système d'activité correspond à des actions qui sont menées à la fois par des individus et par une collectivité. Ces actions sont tributaires des conditions de réalisation (e.g., éléments facilitants et/ou contraignants) et supposent une motivation voire une mobilisation partagée des ressources par tous les acteurs concernés dans la poursuite d'un objectif commun de telle sorte qu'une nouvelle forme d'activité prend forme et fait sens pour tous (Barma, 2008; Engeström, 1987). Un système d'activité se doit d'être considéré comme l'unité d'analyse de base, soit une unité non réductible ou divisible «L'objet de l'activité est constamment en mouvement et ne peut pas être réduit à des visées à court terme. » (Barma, 2008, p.161). L'étude de l'activité poursuivie par les sujets n'est ainsi plus uniquement centrée sur la psychologie d'un individu, mais également sur les interactions entre l'individu et les divers outils (e.g., nouvelle stratégie pédagogique ou moyens de communication) et l'individu et les autres actants d'un système d'activité, ce qui implique une nécessaire considération des rôles et de la division du travail des membres (Engeström, 2001, 2010). Le schéma suivant illustre le système d'activité proposé par Engeström (2010). Celui-ci comprend six pôles en interrelations avec l'individu au centre du système d'activité : sujet, objet, instruments ou artéfacts, communauté, règles et division du travail.

Fig. 1 Triangle de

l'activité

(Engeström, 2010)

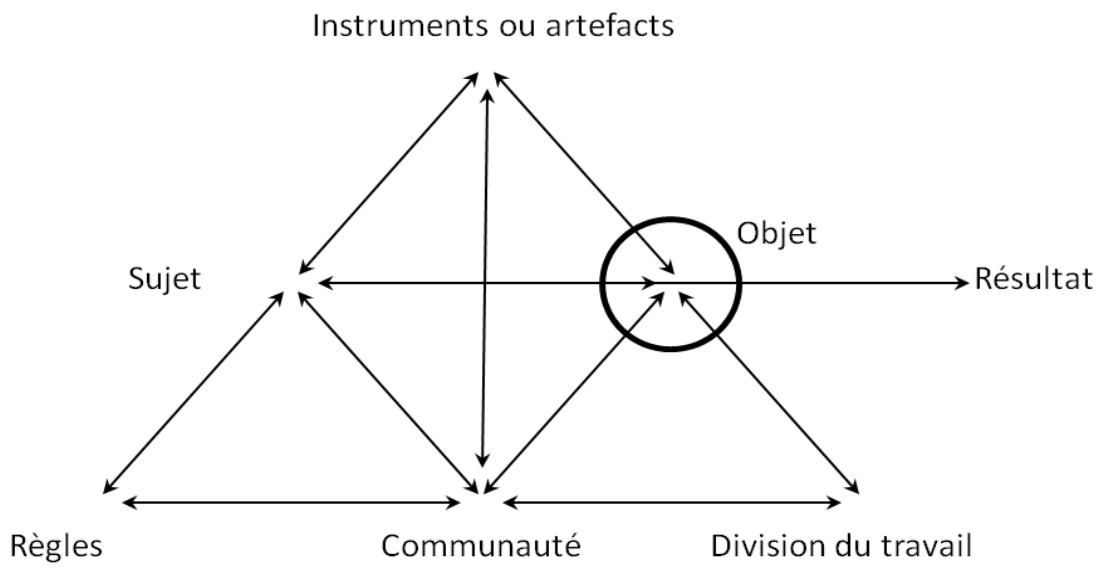

Sur le plan méthodologique, Engeström (1999) précise que son modèle fournit une grille de lecture pour l'analyse de la transformation des pratiques sociales. Deux concepts apparaissent centraux : tensions et contradictions. Les actions individuelles sont souvent en tension avec l'activité du système. Les tensions, si non résolues de part et d'autre, sont génératrices de conflits qui deviennent récurrents. Pour leur part, les contradictions sont cumulées au fil du temps et correspondent à des tensions structurelles à l'intérieur et entre les systèmes d'activité (Virkkunen et Newnham, 2013). Ces contradictions ont le potentiel d'être des moteurs de changement et de développement (Engeström, 1987; Kajamaa, et Schulz, 2014). Somme toute, la théorie de l'activité offre des outils d'analyse permettant de mieux comprendre les dialogues, les perspectives multiples, les contradictions ainsi que les obstacles et les conditions facilitantes susceptibles de les surmonter et de soutenir les innovations (Engeström, 2010; Sannino et Nocon, 2008).

\section{Le Laboratoire du Changement comme méthode d'intervention}

En cohérence avec une épistémologie orientée vers la transformation des pratiques, le Laboratoire du Changement renvoie à un mode d'intervention animé par un intervenant-chercheur et regroupant un petit groupe de participants, employeurs et employés, désireux de comprendre, d'élaborer et d'expérimenter de nouvelles formes de travail dans le cadre d'un ou de plusieurs systèmes d'activité dans lesquels ils œuvrent. Cette méthode suggère que l'équipe de recherche intervention et les acteurs travaillent conjointement pour identifier les problèmes rencontrés et leur 
faire face afin de créer de nouveaux outils en vue de s'affranchir d'une situation problème. Habituellement, le Laboratoire du Changement comporte 5 à 12 rencontres pour 1) analyser et préciser les défis rencontrés dans le milieu et 2) modéliser conjointement des solutions possibles (Engeström, 2001; Virkkunen et Newnham, 2013). Basée sur le cycle d'expansion formulé par Engeström (1987), la méthode du Laboratoire du Changement comporte six phases : 1) questionnement ou état de la situation; 2) analyse de la situation aux plans historico-culturels et empiriques; 3) création d'un nouveau modèle; 4) concrétisation et mise à l'essai du nouveau modèle; 5) implantation d'un nouveau modèle et 6) réflexion et consolidation (Barma, 2014; Virkkunen et Newnham, 2013). Il faut souligner qu'au Québec, cette méthode n'est qu'à l'état embryonnaire. Il faut donc l'explorer davantage et s'approprier cette façon de faire. C'est pourquoi nous avons choisi de nous centrer sur les deux premières phases de cette méthode d'intervention dans l'article actuel. Les deux rencontres sont enregistrées sur bande vidéo. Des études ont montré que l'analyse dialectique des échanges entre participants arrive à montrer les liens entre les manifestations discursives des contradictions et contribue ainsi à donner un sens à l'activité partagée par les acteurs concernés (Sannino, 2008).

\section{Considérations méthodologiques}

La section qui suit présente dans un premier temps quelques résultats provenant d'une étude réalisée auprès d'enseignants œuvrant dans la région de l'école ciblée (Deslandes et Morin, 2015). Puis elle décrit les deux premières rencontres du Laboratoire du Changement. Les données relatives à la première rencontre ont été colligées dans le journal de bord du chercheurintervenant 1 , puis validées auprès des participants. Les données de la deuxième rencontre qui se voulait en continuité avec la première ont été enregistrées sur bande vidéo et retranscrites aux fins d'analyse.

\section{Collecte de données préalable aux interventions}

Préalablement à la première phase de l'intervention, nous avons recueilli des données à l'aide d'entrevues individuelles semi-structurées dans le cadre d'une étude menée auprès de six enseignants œuvrant dans la même région que l'école ciblée (Deslandes et Morin, 2014; Deslandes, Barma et Morin, sous-presse). Ces données ont fait ressortir certains éléments susceptibles d'agir comme conditions contraignantes dans les relations entre les enseignants et les parents. En effet, les résultats mettent en évidence la présence d'une idéologie mercantile de l'école qui s'est implantée dans plusieurs établissements scolaires et qui représente les parents comme des consommateurs. Cette culture de clientélisme semble conférer plus de pouvoir aux parents que par le passé et ouvrir la porte à des attentes et des exigences plus grandes et parfois démesurées de la part de certains parents. Afin de mettre quelques-unes de ces données en contexte, voici quelques extraits qui ont d'ailleurs été utilisés comme matériel miroir lors de la première rencontre.

- Une enseignante déplore : [...] je pense qu'il s'est passé quelque chose en quelque part. Les parents ont pris trop de place dans les écoles. Moi, je trouve qu'ils sont trop présents (P1).

Selon leur avis, les parents en général sont enclins à déléguer beaucoup de responsabilités à l'école et à l'enseignant (P1). Pour s'y intéresser, il faut qu'une situation concerne soit l'enfant ou l'école. À cet effet, un participant déclare :

- $\quad$ Oui, mettre mes limites et prendre ce qui m'appartient et laisser à l'autre personne ce qui lui appartient aussi (P4)

Par ailleurs, selon des participants à l'étude, beaucoup d'enseignants ne veulent pas d'une plus grande communication, d'une plus grande collaboration avec les parents parce que ces derniers surprotègent leur enfant et parce qu'il n'y a pas assez de soutien de la part 
de la direction (P2). Ces données ont fait partie du matériel miroir utilisé au cours des deux premières rencontres du Laboratoire du Changement.

\section{Interventions dans le cadre du Laboratoire du Changement}

Participants. Le groupe de participants, incluant deux membres de l'équipe de recherche intervention $(\mathrm{n}=9)$ et issus d'une même école primaire située en milieu défavorisé, était composé d'une enseignante également présidente du syndicat local, d'un membre de la direction, de deux enseignantes œuvrant à la maternelle et l'autre, en troisième année primaire, de deux parents, soit des mères d'enfants inscrits à l'école primaire ciblée de même que des intervenants-chercheurs (voir Tableau 1). Un membre du syndicat des enseignants à l'échelle provinciale, mais très près du milieu visé, a demandé à être présent à la première rencontre afin de contribuer aux échanges à titre de participant. Faute de temps, il n'a pu être présent à la deuxième rencontre. Les participants, désireux de développer en matière de relations entre les enseignants et les parents, ont donc choisi de participer sur une base volontaire à une démarche de Laboratoire du Changement. Il est ici important de souligner que celle-ci constituait pour eux une toute nouvelle façon de réfléchir collectivement à la problématique école-famille.

Tableau 1 Aperçu des deux rencontres

\begin{tabular}{|c|c|c|c|}
\hline $\begin{array}{l}\text { Dates des } \\
\text { rencontres }\end{array}$ & Durée (h) & Objets des rencontres & $\begin{array}{l}\text { Participants aux deux rencontres } \\
(\mathrm{N}=9)\end{array}$ \\
\hline 28 avril 2014 & 2 heures & $\begin{array}{l}\text { Identification de catégories de } \\
\text { situations irritantes en lien } \\
\text { avec les relations enseignants- } \\
\text { parents }\end{array}$ & $\begin{array}{l}\text { - } 1 \text { enseignante avec un poste au } \\
\text { Syndicat de la région } \\
-\quad 1 \text { membre de la direction d'une } \\
\text { école primaire }\end{array}$ \\
\hline \multirow[t]{2}{*}{5 mai 2014} & 1 heure & $\begin{array}{l}\text { - Échanges sur les tensions } \\
\text { susceptibles d'émerger au } \\
\text { quotidien dans le cadre d'un } \\
\text { panel portant sur les relations } \\
\text { enseignants-parents. }\end{array}$ & $\begin{array}{l}\text { - } 2 \text { enseignantes de la même } \\
\text { école } \\
\text { - } 2 \text { parents ( } 2 \text { mères) d'élèves } \\
\text { fréquentant la même école } \\
\text { - } 2 \text { chercheurs-intervenants }\end{array}$ \\
\hline & & $\begin{array}{l}\text { Amorce d'identification de } \\
\text { solutions. }\end{array}$ & $\begin{array}{l}\text { - } 1 \text { membre du syndicat des } \\
\text { enseignants à l'échelle } \\
\text { provinciale }\end{array}$ \\
\hline
\end{tabular}

Première rencontre du Laboratoire du Changement. Une première rencontre d'une durée de 2 heures a eu lieu dans l'établissement scolaire ciblé après les heures de classe. Les objectifs de cette première rencontre consistaient à : 1) faire d'abord connaissance d'abord, surtout avec l'équipe de recherche intervention, car les autres participants se connaissaient déjà; 2) présenter succinctement la nature du Laboratoire du Changement par l'équipe de recherche intervention; 3) présenter quelques données provenant de l'étude récente menée dans le milieu et 4) amorcer l'identification de zones de tensions entre la direction, les enseignants et les parents. Les données relatives à cette première rencontre ont été colligées dans le journal de bord du chercheur-intervenant 1 , puis validées auprès des participants avant la deuxième rencontre. À la fin de cette dernière, il a été convenu de se pencher sur trois catégories de situations problèmes susceptibles de contribuer à des tensions dans les relations entre les enseignants et les parents : 1) des situations rencontrées dans la quotidienneté des enseignants comme le suivi de conséquences données à un élève ou l'attente de coupons-réponses de la part des parents; 2) a) des demandes individuelles provenant de parents comme celle voulant que son enfant soit placé dans la première rangée de la classe ou celle visant à avoir les devoirs à l'avance à cause de vacances, de tournois, de compétitions à venir et $b$ ) des demandes de l'enseignant à l'endroit des parents comme celle consistant à envoyer le montant exact de l'argent requis pour le lendemain; 3) des démarches de parents adressées directement à la direction avec omission de passer par l'enseignant de l'enfant.

Deuxième rencontre du Laboratoire du Changement. Cette deuxième rencontre a été réalisée deux semaines après la première dans le cadre d'un colloque sur invitation regroupant plusieurs intervenants issus de divers paliers d'intervention du milieu de l'éducation au Québec et mettant à profit un Laboratoire du Changement. Les conversations entre les six participants à ce 
panel en mode labo intitulé La participation des parents (relations enseignants-parents) ont duré 45 minutes. Par la suite, il y a eu des échanges d'une durée de 15 minutes avec les autres participants au colloque qui comprenaient des praticiens, des professionnels de l'éducation, des enseignants, des administrateurs, des chercheurs, des membres d'organisations syndicales, etc. Il faut préciser que ces derniers participants avaient assisté comme audience au panel en mode du Laboratoire du Changement.

\section{Démarche d'analyse et résultats}

Rappelons que deux questions de recherche ont guidé l'analyse des propos tenus lors de ces rencontres : 1) quelles sont les zones de tensions relevées par les participants dans les relations entre les enseignants et les parents? 2) quelles contradictions ont émergé lors des échanges entre les participants?

Ainsi, un premier niveau d'analyse, qualifié de dialectique, a été effectué en lien avec les zones de tensions relevées dans les conversations entre les participants (Engeström et Sannino, 2011). Cette analyse sur les zones de tensions a été réalisée par un groupe de cinq experts en la théorie de l'activité et présents lors de la deuxième rencontre. L'analyse a ensuite été poursuivie par l'intervenant-chercheur 1 à partir des conversations qui avaient été enregistrées puis retranscrites. L'objectif consistait à identifier quelques contradictions qui avaient émergé au cours des échanges entre les participants. Le cadre dialectique traite des systèmes en mouvement à travers le temps. Une contradiction dialectique se réfère à une unité des contraires, des forces opposées, ce qui nous pousse à examiner les verbatims selon cette vision. Le repérage des manifestations discursives selon l'aspect dialectique s'accomplit par une lecture attentive du corpus et une reconnaissance d'éléments indissociables, mais s'opposant à la fois (Engeström, 2010; Virkkunen et Newnham, 2013).

\section{Quelques contradictions identifiées}

Rappelons que les contradictions correspondent à tensions structurelles à l'intérieur et entre les systèmes d'activité. Bien que certaines contradictions génèrent des conflits, elles peuvent néanmoins contribuer à des innovations susceptibles de changer l'activité (Engeström, 2010). D'ailleurs, Engeström et Sannino (2013) présentent comme premier élément de leur modèle d'agentivité transformatrice, l'identification de « situation de motifs contradictoires » (p. 7). Ces auteurs soutiennent que l'analyse et la résolution des contradictions rendent possible le cycle de l'apprentissage expansif et la transformation de l'initiative individuelle vers un effort collectif qui épouse alors un mouvement ascendant de l'abstrait au concret (Engeström, 2013; Engeström et Sannino, 2013).

Quelles sont donc ces contradictions primaires internes, c'est-à-dire à l'intérieur du système d'activité, qui ont été identifiées? Comme proposé par Lapshin, Ivanova et Chernysh (sous-presse) les contradictions internes sont analysées selon chacun des pôles d'un système d'activité (voir figure 2).

Dans le cas des relations entre les enseignants et les parents, la contradiction centrale se situe au niveau de la production de l'objet. D'une part, le milieu scolaire semble vouloir favoriser la participation des parents, mais d'autre part, comme l'affirme un parent : « ... l'ouverture aux parents qui veulent s'impliquer n'est pas grande ». Par ailleurs, lorsqu'elle l'est, cela amène son lot de tensions auprès des enseignants. Y a-t-il une réelle ouverture? Comment les acteurs (enseignants et directions/parents) définissent-ils la participation parentale? Quelles sont les attentes de participation au suivi scolaire selon la compréhension des deux principaux groupes d'acteurs de cette même école? 


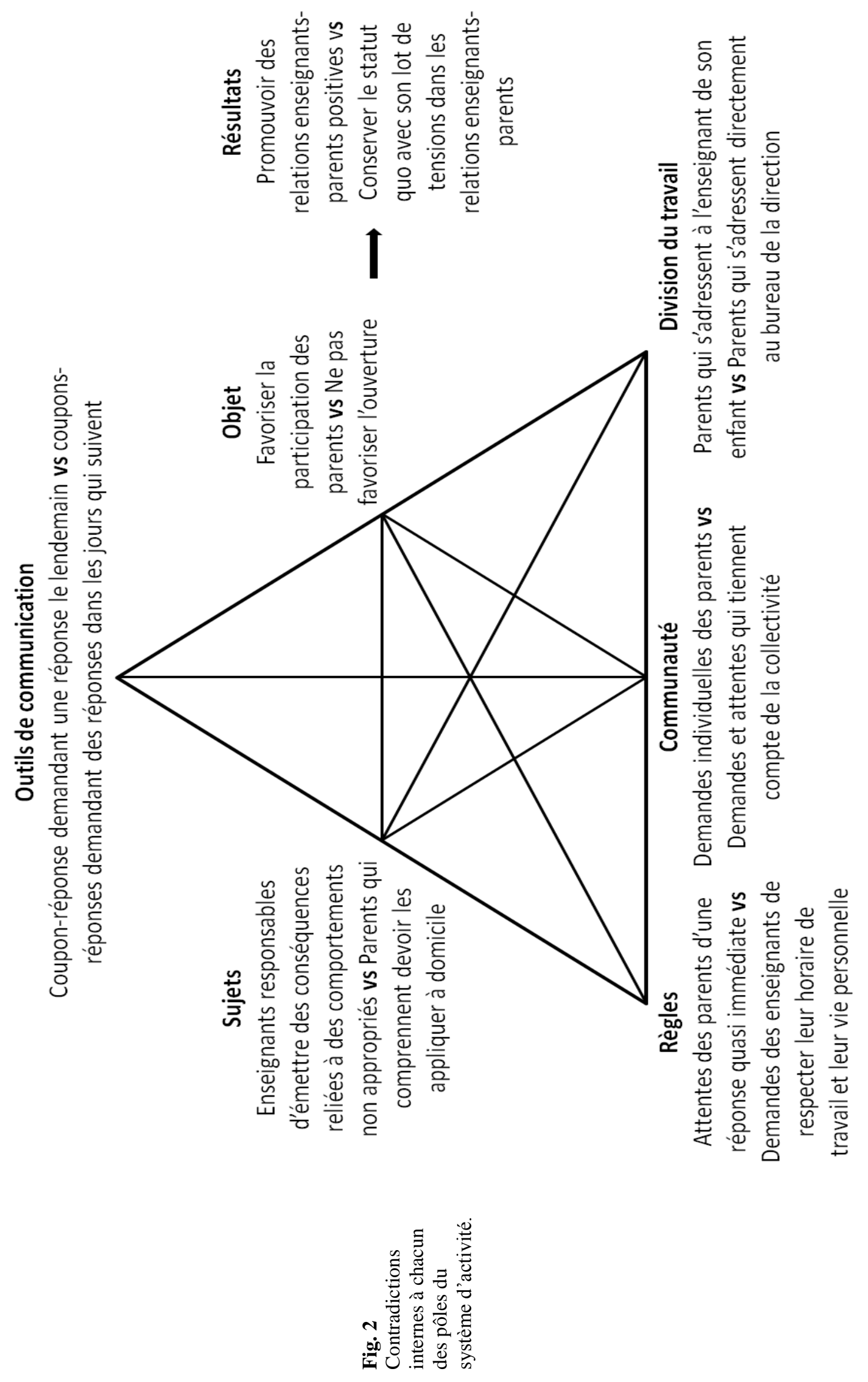


Une deuxième contradiction interne se retrouve au plan de l'outil. Le milieu scolaire se veut clair et transparent dans ses communications, mais certains messages (coupons-réponses) ne correspondent pas vraiment à l'idée que l'on veut véhiculer. Un membre de la direction dit : « On demande pour le lendemain, mais ce n'est pas vraiment cela... Enseignant : on demande de le rapporter le lendemain pour l'avoir d'ici quelques jours. Souvent, il faut envoyer un 2e envoi. » Pourquoi alors ne pas le dire clairement? Il se peut que l'outil utilisé pour communiquer ne soit pas adéquat, voire suffisamment clair et précis pour tous les parents.

Une troisième contradiction a trait à la division du travail. Des parents s'attendent à des réponses de la part de l'enseignant dans la même journée. Un enseignant dit : « Des parents écrivent le matin à $9 \mathrm{~h}$; à midi, s'ils n'ont pas eu de réponse, ils écrivent à la direction... la technologie peut devenir lourde...». Un autre enseignant déclare : «Les enseignants veulent améliorer la communication, mais ils ont aussi une vie familiale, personnelle... » Que doit-on comprendre de cette situation? Il est possible que ce soit attribuable à un manque de compréhension des éléments du contexte de vie personnelle et professionnelle de l'enseignant. De même, il est plausible que ce soit associé à un manque de disponibilité des parents ou un manque de respect ou encore une méconnaissance de l'aspect multitâches d'un professionnel en enseignement. Voilà autant d'explications qui pourront être présentées à titre de données miroir lors d'une rencontre ultérieure avec les participants afin de coconstruire une compréhension commune de la situation problème et d'envisager des façons de dépasser les contradictions identifiées.

Une quatrième contradiction interne concerne les règles de fonctionnement établies dans l'école. Ainsi, les parents qui n'ont pas de réponses immédiates de la part de l'enseignant « écrivent à la direction » selon une participante enseignante. Or, dans les écoles, il est généralement habituel de s'adresser d'abord à l'enseignant lorsqu'il est question de son enfant. Peut-être que cette règle parfois implicite mériterait d'être davantage expliquée aux parents dès le début de l'année scolaire. Autrement dit, il serait pertinent de rappeler aux parents que la personne à contacter en premier lieu, est l'enseignant et que ce dernier, comme responsable d'un groupe classe, n'a pas nécessairement la disponibilité pour répondre aux courriels des parents dans un court laps de temps.

Une cinquième contradiction, reliée au pôle sujets (voir figure 2) révèle que les parents se sentent responsables de donner suite à une démarche entreprise par l'enseignant. Un parent dit : «On reçoit des codes jaunes ou rouges à la maison. Parfois, des parents ne savent pas ce que ça veut dire. Mais on doit soutenir l'équipe-école ». Un enseignant précise : «À la première rencontre de parents, on vous écrit sur ce qui est attendu. Les conséquences sont appliquées à l'école. On ne s'attend pas à ce qu'elles soient appliquées à la maison ». Cette contradiction gagnerait à être présentée aux deux groupes d'acteurs comme autre donnée miroir lors d'une prochaine rencontre du Laboratoire du Changement afin de laisser émerger des propositions d'actions concrètes pour remédier à cette incompréhension.

Les autres contradictions internes identifiées se situent au plan de la communauté. Elles illustrent des demandes de parents sur le plan individuel au détriment de l'ensemble des autres parents de la collectivité. On sait que la très grande majorité des parents désirent que l'enseignant stimule et favorise les apprentissages des élèves, mais certains parents demandent : « Pouvez-vous rien faire d'important ....telle journée, car mon enfant devra s'absenter? » pour un rendez-vous chez le dentiste ou chez le médecin, par exemple. D'autres parents « demandent du travail en avance pour leur enfant » parce qu'ils vont en voyage ou encore parce que leur enfant participe à un tournoi de hockey. Ces deux situations illustrent une perspective individualiste en opposition avec une perspective collective que l'enseignant doit adopter pour son groupe d'élèves. Elles soulèvent aussi des enjeux sociétaux qui semblent être en opposition avec les visées de l'école : comment expliquer que des professionnels de la santé n'offrent pas de disponibilité le soir alors que la société réclame haut et fort un plus haut taux de réussite et de persévérance scolaires?

\section{Quelques zones de tension}

Selon Engeström et Sannino (2011), quatre zones de tensions axées sur des critères linguistiques sont possibles : des doubles-contraintes, des conflits critiques, des conflits et des dilemmes. La double-contrainte signifie que les individus doivent faire face à des alternatives tout 
aussi non-gagnantes dans un cas comme dans l'autre. Le conflit critique renvoie à des motifs contradictoires dans l'interaction sociale, comme avoir le sentiment d'être violé ou coupable. Le conflit est synonyme d'argument, de critique tandis que le dilemme nous dirige vers des expressions ou des échanges d'évaluations incompatibles (traduction libre, Barma 2014, voir Tableau 2). Les analyses qui suivent se sont donc inspirées des travaux de Engeström et Sannino (2011).

Tableau 2 Types de manifestations discursives de contradictions et exemples tirés de verbatims

\begin{tabular}{|c|c|c|c|}
\hline Manifestations & $\begin{array}{l}\text { Nombre de } \\
\text { manifestations } \\
\text { relevées }\end{array}$ & Caractéristiques & Exemples tirés de verbatims \\
\hline $\begin{array}{l}\text { Double } \\
\text { contrainte }\end{array}$ & 5 & $\begin{array}{l}\text { Faire face à des } \\
\text { alternatives tout } \\
\text { aussi non- } \\
\text { gagnantes dans un } \\
\text { cas comme dans } \\
\text { l'autre }\end{array}$ & $\begin{array}{l}\text { Enseignant : on demande de le rapporter le } \\
\text { lendemain pour l'avoir d'ici quelques } \\
\text { jours. Souvent, il faut envoyer un } 2 \mathrm{e} \text { envoi, } \\
\text { puis un } 3 \mathrm{e} \text { et ce sont souvent les mêmes... } \\
\text { Parent : Parfois, on demande le montant } \\
\text { exact }(2,60 \text { \$) dans une enveloppe pour le } \\
\text { lendemain matin. }\end{array}$ \\
\hline Conflit critique & 0 & $\begin{array}{l}\text { Faire face à des } \\
\text { motifs } \\
\text { contradictoires } \\
\text { dans l'interaction } \\
\text { sociale, avoir le } \\
\text { sentiment d'être } \\
\text { violé ou coupable; } \\
\text { émotivité }\end{array}$ & \\
\hline Conflit & 2 & $\begin{array}{l}\text { Argument, } \\
\text { critique }\end{array}$ & $\begin{array}{l}\text { Parent :... si le parent va à la Direction : ça } \\
\text { devient menaçant pour le prof ». } \\
\text { Parent : Parfois, on est plus en contact } \\
\text { avec la Direction, on la connaît plus. }\end{array}$ \\
\hline Dilemme & 5 & $\begin{array}{l}\text { Expression ou } \\
\text { échange } \\
\text { d'évaluations } \\
\text { incompatibles. }\end{array}$ & $\begin{array}{l}\text { Enseignant : Il y a beaucoup de messages } \\
\text { comme n'oubliez pas d'envoyer mon } \\
\text { enfant au poste... car il a un rendez-vous } \\
\text { chez le médecin. } \\
\text { Enseignant : Des parents vont dans le Sud, } \\
\text { en voyage, à des tournois de hockey; des } \\
\text { parents demandent quel travail ils devront } \\
\text { faire }\end{array}$ \\
\hline
\end{tabular}

Pour ces auteurs, les attentes non exprimées, non précisées ou tout simplement méconnues constituent l'une des principales sources de tensions au sein des relations entre les enseignants et les parents. Dans le cas de cette recherche-intervention, les attentes des enseignants à l'égard des parents ont trait à la réalisation des devoirs, aux coupons-réponses, à l'argent à remettre le plus tôt possible aux enseignants et à la prise en compte par les parents, de leur réalité. Pour ce qui est des devoirs, une enseignante mentionne : «On s'interroge sur la pertinence des devoirs et leçons. Il y en a 4 (élèves) qui font leurs devoirs à l'aide aux devoirs, 6-7 sur l'heure du midi et 5-6 qui ne les font pas. Ils les font alors en retenue ». Voici un exemple de tensions exprimées sous la forme d'une double contrainte. Sans être explicite, l'enseignant manifeste un désappointement face à l'absence de suivi de certains parents dans le cadre des devoirs. D'après l'enseignant, une transformation pratique allant au-delà des mots s'avère nécessaire pour remédier à la problématique des devoirs et leçons. À cet effet, un membre de la direction de l'école déclare : «Le comité va réfléchir sur les devoirs et leçons. Dans le cadre du projet éducatif, des questionnaires ont été envoyés aux parents ». L'école et les enseignants s'attendent à ce que les parents exercent un suivi des devoirs et leçons (Deslandes, 2009). Cependant, à la lumière des propos de l'enseignante, il semble que ce ne soit pas le cas. 
Quant aux coupons-réponses, d'un côté, les enseignants demandent de les rapporter le lendemain pour les avoir avant quelques jours et se plaignent de devoir le réclamer jour après jour tandis qu'un des parents déplore que l'on demande le montant exact de l'argent pour le lendemain matin. Par ailleurs, un membre de la direction précise : «On demande pour le lendemain, mais ce n'est pas vraiment cela....». Il s'agit ici de tensions manifestées sous forme de double contrainte exprimant des situations incompatibles entre les obligations des enseignants et des éléments du contexte de vie des parents. Les explications proposées par des participants lors de la deuxième rencontre sont associées à un manque de communication et un manque de connaissance de la réalité quotidienne de l'enseignant et de ses responsabilités professionnelles.

- Parent : Il y a un manque de communication; ce ne sont pas tous les parents qui peuvent aller à la rencontre en début d'année, des petits détails sont manquants; ce n'est pas nécessairement un manque de bonne volonté.

- Enseignant : L'enseignant a 27-28 élèves dans sa classe. Il ne peut pas répondre aux différentes demandes de tous les parents...

De même, un enseignant aimerait que le parent prenne en compte le contexte de l'exercice de sa profession avec un grand nombre d'élèves en classe en même temps et le manque de disponibilité pour répondre à des messages téléphoniques ou des courriels pendant les heures de classe. Un enseignant dit : "Des parents écrivent le matin à $9 \mathrm{~h}$; à midi, s'ils n'ont pas eu de réponse, ils écrivent à la Direction... la technologie peut devenir lourde... ». Voilà une tension exprimée sous forme de dilemme.

D'autres tensions sous forme de dilemme sont exprimées sous l'angle des attentes parentales. Ces attentes à l'égard de l'enseignant ont trait à des demandes particulières et individuelles des parents comme préparer l'enfant et son sac pour $14 \mathrm{~h}$, car il a un rendez-vous chez le dentiste ou le médecin, ou lui donner du travail à effectuer pendant une semaine de vacances familiales ou encore le placer dans la première rangée de la classe.

- Enseignant : Il y a beaucoup de messages comme n'oubliez pas d'envoyer mon enfant au poste... car il a un rendez-vous chez le médecin.

- Enseignant : Des parents vont dans le Sud, en voyage, à des tournois de hockey; des parents demandent quel travail ils devront faire.

De même, d'autres tensions sous forme de double contrainte sont aussi relevées sous l'égide des attentes parentales. Elles sont en lien avec la clarté des informations qui lui sont rapportées lorsqu'un enfant reçoit un code jaune ou un code rouge à l'école. Règle générale, un code jaune signifie que l'élève a fait un manquement au code de vie. L'information, qui se veut un avertissement, est envoyée aux parents souvent par le billet de l'agenda. Un code rouge correspond à un comportement grave qui est susceptible de nécessiter une intervention de la direction. Un parent déclare : «On reçoit des codes jaunes ou rouges à la maison. Parfois, des parents ne savent pas ce que ça veut dire, mais on doit soutenir l'équipe-école ». Par ailleurs, un enseignant dit que toutes les explications sont données en début d'année : «À la première rencontre de parents, on parle de sur ce qui est attendu. Les conséquences sont appliquées à l'école. On ne s'attend pas à ce qu'on les applique à la maison ».

À la possibilité de s'impliquer dans l'école, un parent mentionne : "Quand on fait du bénévolat, ça aide à créer des liens positifs avec les enseignants et les autres enfants ». Un peu plus tard, ce même parent affirme en parlant des enseignants : «l'ouverture aux parents qui veulent s'impliquer n'est pas grande », exprimant ainsi une tension sous forme de dilemme. Plusieurs explications à ces tensions sont également mises de l'avant. Le manque de communication y trouve sa pertinence une fois de plus. S'ajoute une perspective individualiste de la part des parents et ce, au détriment des autres élèves en tant que groupe. En d'autres termes, certains parents ne pensent qu'à l'intérêt de leur propre enfant, oubliant par le fait même les intérêts des autres élèves de son groupe-classe. 
Des parents ignorent l'enseignant qui est en classe et s'adressent directement à la direction, la jugeant plus accessible. Un enseignant dit : «L'enseignant est frustré : quel message le parent veut-il passer? » De façon subtile, cet enseignant exprime la présence de tensions sous forme de conflit dans le sens que cette façon d'agir introduit des doutes chez l'enseignant Un parent dit : «... si le parent va à la direction : ça devient menaçant pour le prof ». Un autre parent précise : «Parfois, on est plus en contact avec la direction, on la connaît plus ». Et un membre de la direction ajoute : «C'est à cause du facteur temps. Le parent pressé va vers la direction, car l'enseignant est en classe ». Ce sont ici des tensions manifestées sous forme de dilemme exprimé en termes d'hésitation entre deux choix. Comme autre explication, un parent révèle : "Certains parents veulent du soutien de la direction, car ils ne veulent pas confronter l'enseignant». Le parent met ainsi en évidence une zone de tension sous forme de conflit. Bref, que ce soit sous l'angle d'un manque de temps, d'un manque de disponibilité tant de la part des enseignants que des parents ainsi que d'un manque de soutien parfois de la part de l'enseignant ou d'un manque de confiance à son égard, voilà autant d'éléments qui figurent parmi les explications proposées par les participants en regard des zones de tension dans les relations entre les enseignants et les parents. L'analyse des verbatims de la deuxième rencontre a permis d'identifier autant de manifestations discursives de doubles contraintes $(\mathrm{n}=5)$ que de dilemmes $(\mathrm{n}=5)$ avec un nombre moindre d'expressions de conflits $(n=2)$. Nous n'avons relevé aucune manifestation de conflit critique.

\section{Conclusion}

Les recherches effectuées au cours des dernières décennies ont mis en lumière l'importance de relations entre les enseignants et les parents positives et collaboratrices. Or, un des rôles impartis aux enseignants est de promouvoir la participation parentale au suivi scolaire créant ainsi un terrain fertile en matière de tensions. La présente recherche intervention prend assise sur la théorie de l'activité qui s'intéresse à la transformation des pratiques sociales. Elle met à profit la méthode du Laboratoire du Changement, une méthode d'intervention comportant six phases et regroupant des intervenants chercheurs et des participants désireux d'expérimenter de nouvelles formes de travail dans le cadre d'au moins un système d'activité dans lequel ils œuvrent. Cette étude comporte des limites. Soulignons que seulement les deux premières phases de l'intervention qui en comprend habituellement six sont analysées dans l'article actuel. Une autre limite a trait à l'impossibilité de généraliser les résultats, car l'école ciblée, à l'instar de tout autre établissement scolaire, affiche ses propres spécificités et ses propres situations problèmes.

L'analyse des deux premières rencontres a permis d'identifier les zones de tensions et de contradictions primaires au sein des relations enseignants-parents d'une même école et de les positionner en fonction de chacun des pôles du triangle du système d'activité. La principale contradiction se retrouve au plan de l'objet et questionne la motivation des enseignants à promouvoir ou non la participation parentale et de façon particulière à l'école. Il semble que malgré les résultats d'études portant sur les retombées positives de la participation parentale (Deslandes, 2010; Epstein, 2011), on en soit encore à se demander si la participation parentale est véritablement souhaitée. Or, la recherche a montré que « «l'école se doit d'envoyer des messages constants à tous les parents exprimant que leur participation est à la fois désirée et nécessaire pour soutenir les jeunes en tant qu'apprenants » (Deslandes, 2015, p.211). Il apparaît impérieux de rapporter cette contradiction comme donnée miroir lors des rencontres suivantes du Laboratoire du Changement pour mettre au clair la compréhension et les attentes des deux groupes d'acteurs et d'identifier des pistes de solution.

D'autres contradictions sont identifiées en matière de communication, outil névralgique à la base de relations positives entre les enseignants et les parents. Au plan de l'outil, certaines communications entre enseignants et parents ne semblent pas suffisamment claires et précises. Nombreux sont les travaux ayant démontré que les communications entre l'école et les parents se doivent d'être ouvertes, claires, régulières et utiliser un langage facilement accessible (Deslandes, 2006, 2010; Epstein, 2011). Une contradiction a aussi été identifiée sous l'égide de la division du travail en termes d'attentes irréalistes des parents au regard de réponses quasi immédiates à leurs demandes d'une part et d'autre part, des enseignants qui réclament la prise en compte de leur vie professionnelle et personnelle. Cette contradiction de même que celle relevée en matière de règle 
de fonctionnement où des parents contactent directement la direction au lieu de s'adresser en premier lieu à l'enseignant font référence à des savoir-faire et des savoir-être qui reposent sur le respect, la confiance mutuelle et un partage des responsabilités (Deslandes, 2006, 2012). Finalement, les contradictions au plan de la communauté, tel qu'illustré dans des études antérieures (Deslandes, 2012; Deslandes et al., sous-presse) nous renvoient à la nature mercantile et individualiste des rapports entre certains parents et l'école.

L'analyse des deux premières rencontres entre des enseignants et des parents a permis de jeter les premiers jalons d'une démarche de Laboratoire du Changement. Sur le plan méthodologique, il est impérieux de continuer l'exploration des phases subséquentes d'un Laboratoire du Changement. Au plan de la recherche, il est souhaitable d'examiner éventuellement les expressions d'agentivité transformatrice susceptibles de révéler comment une idée ou une suggestion a été développée par les participants et de quelles manières elles ont évolué lors d'une même rencontre. Partant du cycle d'expansion élaboré par Engeström (1987) la prochaine étape dans la poursuite du Laboratoire du Changement consiste à présenter aux participants l'analyse dialectique réalisée par les chercheurs et, en cohérence avec la méthode du Laboratoire du Changement, de la réinvestir sous forme de miroir aux participants. Sur cette base, le chercheurintervenant l'utilise comme un tremplin pour valider l'identification du problème, en discuter et s'engager dans une comodélisation d'une nouvelle forme de collaboration entre les enseignants et les parents.

\section{Références}

Barma, S. (2008). Un contexte de renouvellement de pratiques en éducation aux sciences et aux technologies : une étude de cas réalisée sous l'angle de la théorie de l'activité. (Thèse de doctorat). Récupéré de http://www.theses.ulaval.ca/2008/25695/.

Barma, S. (2012). La théorie de l'activité : un tour d'horizon. Vers une grille de lecture socioculturelle pour interpréter le renouvellement des pratiques en éducation. Présenté dans le cours DID-7025, Québec, Université Laval, 12 p.

Barma, S. (2014). Mot de la rédaction : Le CRIRES et l'innovation sociale en éducation. Revue internationale du CRIRES : innover dans la tradition de Vygotsky, 2(1), 1-6.

Caspe, M., Lopez, M. E., \& Wolos, C. (2007). Family Involvement in Elementary School Children's Education. Family Involvement Makes a Difference: Evidence that Family Involvement Promotes School Success for Every Child of Every Age. Harvard Family Research Project. 2 (Winter 2006/2007) Récupéré : http://www.hfrp.org/publications-resources/publications-series/family-involvement-makesa-difference/family-involvement-in-elementary-school-children-s-education

Christenson, S. L. \& Sheridan, S. M. (2001). Schools and families: Creating essential connections for learning. New-York, N.Y. : The Guilford Press.

Deslandes, R. (2001). L'environnement scolaire. Dans M. Hamel, L. Blanchet et C. Martin (dirs), 6-12-17, nous serons bien mieux! Les déterminants de la santé et du bien-être des enfants d'âge scolaire (p. 251286), Québec : Les Publications du Québec.

Deslandes, R. (2005). Réussite scolaire : déterminants et impact des relations entre l'école et la famille. Dans L. Deblois et D. Lamothe (dir.), La réussite scolaire. Comprendre et mieux intervenir (p. 223236), Québec : Presses de l'Université Laval.

Deslandes, R. (2006). La problématique école-famille-communauté dans la formation des maîtres. Dans J. Loiselle, L. Lafortune et N. Rousseau (dir.), L'innovation et la formation à l'enseignement : pistes de réflexion et d'action pour les futurs enseignants (p. 183-205), Québec : Presses de l'Université du Québec.

Deslandes, R. (2007). Rôle de la famille, liens école-famille et résilience scolaire. Dans J. P. Pourtois, B. Cyrulnik et P. Duval (dirs.). École et résilience (p. 270-295), Paris : Odile Jacob.

Deslandes, R. (2008). Analyse d'une recherche-action sur la collaboration école-famille-communauté. Dans R. Deslandes (Dir.) : La collaboration de l'école, de la famille et de la communauté à l'apprentissage. Montréal, Québec : Association canadienne-française pour l'avancement des sciences (ACFAS).

Deslandes, R. (2009). International perspectives on student outcomes and homework : Family-schoolcommunity partnerships. New York (USA), London (UK) and Canada : Routledge. 
Deslandes, R. (2010). Le difficile équilibre entre la collaboration et l'adaptation dans les relations écolefamille. Dans G. Pronovost (dir.), Familles et réussite éducative (p. 197-215), Actes de colloque du 10e Symposium québécois de Recherche sur la famille, Québec : PUQ.

Deslandes, R. (2011). Les relations école-famille-communauté au cour des apprentissages et du développement des jeunes. RIRE-CTREQ, Récupéré : http://rire.ctreq.qc.ca/2011/02/les-relations-ecolefamille-communaute-au-coeur-des-apprentissages-et-du-developpement-des-jeunes/

Deslandes, R. (2012). Un modèle du développement humain au service de la réussite éducative du jeune : vers un modèle intégrateur des facteurs et processus de la collaboration école-famille, Revue Développement humain, handicap et changement social, 20(3), 77-92.

Deslandes, R. (2013). La collaboration parent-enseignante au regard de l'état actuel des connaissances. Revue préscolaire, 51(4), 11-14.

Deslandes, R. (2015, 3e éd.). Collaboration famille-école-communauté pour une inclusion réussie. Dans N. Rousseau (dir.), La pédagogie de l'inclusion scolaire (p.203-232). Québec : Presses de l'Université du Québec

Deslandes, R. \& Bertrand, R. (2001). La création d'une véritable communauté éducative autour de l'élève : une intervention plus cohérente et des services mieux harmonisés. CRIRES : Rapport de recension des écrits. Récupéré : http://crires.ulaval.ca/financement/la-creation-dune-veritable-communauteeducative-autour-de-leleve-une-intervention-plus

Deslandes, R. \& Bertrand, R. (2004). Motivation des parents à participer au suivi scolaire de leur enfant au primaire. Revue des sciences de l'éducation, 30(2), 411-434.

Deslandes, R. \& Bertrand, R. (2005). Parent involvement in schooling at the secondary level: Examination of the motivations. The Journal of Educational Research, 98(3), 164-175.

Deslandes, R., Barma, S. \& Morin, L. (2015, sous-presse). Understanding complex relationships between teachers and parents. International Journal about Parents in Education.

Deslandes, R., Bastien, N. \& Lemieux, A. (2006). Programme de partenariat école-famille-communauté. Rapport de recherche remis au Ministère de l'Éducation, du Loisir et du Sport, 260p.

Deslandes, R. \& Morin, L. (2015). Les relations enseignants-parents dans toute leur complexité. Rapport de recherche présenté au Syndicat des enseignants de la Mauricie-Centre du Québec. 46 pages

Engeström, Y. (1987). Learning by expanding: An activity-theoretical approach to developmental research. Helsinki : Orienta-Konsultit.

Engeström, Y. (2010). Expansive learning at work: Toward an activity theoretical reconceptualization. Journal of Education and Work, 14(1), 133-156.

Engeström, Y. \& Sannino, A. (2011). Discursive manifestations of contradictions in organizational change efforts. A methodological framework. Journal of Organizational Change Management 24(3), 368-387.

Engeström, Y., \& Sannino, A. (2013). La volition et l'agentivité transformatrice : perspective théorique de l'activité. Revue internationale du CRIRES : innover dans la tradition de Vygotsky, 1(1), 4-19.

Epstein, J. L. (2011). School, family, and community partnerships: Preparing educators and improving schools, Boulder, Colorado: Westview Press.

Hoover-Dempsey, K. V., Ice, C. L., \& Whitaker, M. C. (2010). Motivation and commitment to familyschool partnerships. In S. L. Christenson \& A. L. Reschly (Eds), Handbook on school-family partnerships for promoting student competence (p.30-60). New York, N. Y. : Routledge, Taylor and Francis Group.

Hoover-Dempsey, K. V. \& Sandler, H. M. (1997). Why do parents become involved in their children's education? Review of Educational Research, 67(1), 3-42.

Kajamaa, A., \& Schulz, K.-P. (2014, July). Organizational diffusion of change, Learning and development : A case example from home care. In Engeström, Y., Kajamaa, A. \& Bodrožić, Z. Activity Theory and Organizations. Symposium conduct at the $30^{\text {th }}$ Colloquium of European Group for Organizationala Studies (EGOS), Rotterdam The Netherlands.

Kreider, H., Caspe, M., Kennedy, S., \& Weiss, H. (2007). Family Involvement in Middle and High School Students' Education. Involvement Makes a Difference : Evidence that Family Involvement Promotes School Success for Every Child of Every Age. Harvard Family Research Project. 3(Spring2007). Retrieved from http://www.hfrp.org/family-involvement/publications-resources/family-involvementin-middle-and-high-school-students-education

Lapshin, Y., Ivanova, A., \& Chernysh, A. (in press). Developing agentive subjects in school : A russian case study of educational change from below. In B Selau, R. F. Castro (Orgs.), Cultural-Historical Approach : Educational research in different contexts (p. 192-212). Porto Alegre : EDIPUCRS

Leont'ev, A. N. (1978). Activity, consciousness, and personality. Englewood Cliffs, NJ: Prentice-Hall.

Sannino, A. (2008). From talk to action : Experiencing interlocution in developmental interventions. Mind, Culture, and Activity, 15(3), 234-257. 
Vygotsky, L. S. (1978). Mind in society: The psychology of higher mental functions. Cambridge, MA : Harvard University Press.

Virkkunen, J., \& Newnham, D. S. (2013). The Change Laboratory. A tool for collaborative development of work and education. Rotterdam : the Netherlands: SensePublishers 